\title{
Frequency of MELAS main mutation in a phenotype-targeted young ischemic stroke patient population
}

\author{
Turgut Tatlisumak $^{1,2,3} \cdot$ Jukka Putaala $^{3} \cdot$ Markus Innilä $^{4} \cdot$ Christian Enzinger $^{5}$ • \\ Tiina M. Metso ${ }^{3}$ - Sami Curtze ${ }^{3}$ - Bettina von Sarnowski ${ }^{6}$ - Alexandre Amaral-Silva ${ }^{7,8}$. \\ Gerhard Jan Jungehulsing ${ }^{9}$ Christian Tanislav ${ }^{10}$ - Vincent Thijs ${ }^{11}$. \\ Arndt Rolfs $^{12}$ • Bo Norrving ${ }^{13}$ - Franz Fazekas ${ }^{5}$ Anu Suomalainen ${ }^{3,4}$. \\ Edwin H. Kolodny ${ }^{14}$
}

Received: 27 June 2015/Revised: 1 November 2015/ Accepted: 2 November 2015/Published online: 14 November 2015 (c) Springer-Verlag Berlin Heidelberg 2015

\begin{abstract}
Mitochondrial diseases, predominantly mitochondrial encephalomyopathy, lactic acidosis, and strokelike episodes (MELAS), may occasionally underlie or coincide with ischemic stroke (IS) in young and middleaged individuals. We searched for undiagnosed patients with MELAS in a target subpopulation of unselected young IS patients enrolled in the Stroke in Young Fabry Patients study (sifap1). Among the 3291 IS patients aged 18-55 years recruited to the sifap1 study at 47 centers across 14 European countries, we identified potential MELAS patients with the following phenotypic features: (a) diagnosed cardiomyopathy or (b) presence of two of the three following findings: migraine, short stature $(\leq 165 \mathrm{~cm}$ for males; $\leq 155 \mathrm{~cm}$ for females), and diabetes. Identified patients' blood samples underwent analysis of the common MELAS mutation, m.3243A $>\mathrm{G}$ in the MTTL1 gene of
\end{abstract}

Turgut Tatlisumak

turgut.tatlisumak@neuro.gu.se; turgut.tatlisumak@hus.fi

1 Institute of Neuroscience and Physiology, Sahlgrenska Academy, University of Gothenburg, Per Dubbsgatan 14, POB 430, 40530 Gothenburg, Sweden

2 Department of Neurology, Sahlgrenska University Hospital, Gothenburg, Sweden

3 Department of Neurology, Helsinki University Central Hospital, Helsinki, Finland

4 Research Program for Molecular Neurology, University of Helsinki, Helsinki, Finland

5 Department of Neurology, Medical University of Graz, Graz, Austria

6 Department of Neurology, Ernst-Moritz-Arndt University Medicine, Greifswald, Germany

7 Department of Neurology, Unidade Cerebrovascular Hospital de São José, Lisbon, Portugal mitochondrial DNA. Clinical and cerebral MRI features of the mutation carriers were reviewed. We analyzed blood samples of 238 patients (177 with cardiomyopathy) leading to identification of four previously unrecognized MELAS main mutation carrier-patients. Their clinical and MRI characteristics were within the expectation for common IS patients except for severe hearing loss in one patient and hyperintensity of the pulvinar thalami on T1-weighted MRI in another one. Genetic testing for the m.3243A $>$ G MELAS mutation in young patients with IS based on phenotypes suggestive of mitochondrial disease identifies previously unrecognized carriers of MELAS main mutation, but does not prove MELAS as the putative cause.

Keywords Mitochondrion - Ischemic stroke · Young · Diagnosis $\cdot$ MELAS $\cdot$ Mitochondrial disease $\cdot$ Mutation

8 Department of Neurology, Hospital Vila Franca de Xira, Vila Franca de Xira, Portugal

9 Department of Neurology, Charite University, Berlin, Germany

10 Department of Neurology, University of Giessen, Giessen, Germany

11 Austin Health and Florey Institute of Neuroscience and Mental Health, University of Melbourne, Heidelberg, VIC, Australia

12 Albrecht-Kossel-Institute for Neuroregeneration, University of Rostock, Rostock, Germany

13 Department of Clinical Neurosciences Neurology, Lund University, Lund, Sweden

14 Department of Neurology, New York University School of Medicine, New York, NY, USA 


\section{Introduction}

Mitochondrial diseases are a heterogeneous group of disorders caused by the dysfunction of the mitochondrial respiratory chain, the essential common pathway for aerobic ATP production. Therefore, tissues and organs highly dependent on oxidative ATP production, such as brain, muscle, and heart, are preferentially affected. An incidence of approximately $1-2$ cases per 10,000 individuals ranks them amongst the most common metabolic diseases. They may manifest at any age, with a wide variability of manifestations [1]. Generally, nuclear DNA abnormalities leading to mitochondrial dysfunction manifest in childhood and mitochondrial DNA (mtDNA) abnormalities in late childhood and adulthood. The resulting multisystem disease presents most commonly with neurological and myopathic features. Mitochondrial encephalomyelopathy with lactic acidosis and stroke-like episodes (MELAS) is the most common mtDNA disease presenting with phenotypic features including short stature, exercise intolerance, neurosensory hearing loss, migraine-like headaches, seizures, myopathy, ophthalmoplegia, cardiomyopathy, axonal neuropathy, diabetes mellitus, pigmentary retinopathy, and renal tubular acidosis [2]. Approximately $80 \%$ of all MELAS patients carry a single mutation of m.3243A $>\mathrm{G}$ in the tRNA ${ }^{\text {leu (UUR) }}$ (MTTL1) within the mtDNA [3].

Mitochondrial diseases are a rare, but well-recognized cause of ischemic stroke (IS)-like episodes at various ages $[2,4]$. While MELAS is the most common cause, other mitochondrial diseases such as myoclonic epilepsy with ragged red fibers (MERRF), Kearns-Sayre syndrome, Leigh syndrome, mitochondrial recessive ataxia syndrome (MIRAS), and hyperornithinemia-hyperammonemia-homocitrullinuria (triple-H syndrome) have also rarely been reported to be associated with IS-like lesions $[4,5]$. The pathophysiology and course of stroke-like episodes in patients with mitochondrial diseases are distinct from IS and are probably underlied by a number of mechanismsalone or in combination-discussed in detail elsewhere [4]. We previously reported only two MELAS patients among 1008 young patients with first-ever IS at their age of 15-49 years [6]. Mitochondrial disease was reported in 4 out of 104 Saudi children presenting with stroke [7]. Recently, 6 patients with mitochondrial disease out of 3331 European young first-ever IS patients were reported by us [8]. However, mitochondrial disease is likely underdiagnosed among IS patients and no systematic targeted or unselected screening approach has been performed.

In the present work, we aimed at detecting undiagnosed MELAS main mutation $(\mathrm{m} .3243 \mathrm{~A}>\mathrm{G})$ carrier-patients in a large multinational European database of a relatively young (18-55 years of age at the index IS onset) population of IS patients recruited to the sifap1 study. We expected undiagnosed MELAS patients to be discovered at a relatively low cost if a phenotype-targeted approach taking into account "red flags" for mitochondrial disease, (e.g., cardiomyopathy, migraine, diabetes, and short stature) would be utilized for phenotypic prescreening before genetic analyses.

\section{Patients and methods}

\section{Patients}

The Stroke in Young Fabry Patients (sifap1) is a European multicenter prospective observational cohort study of white patients aged 18-55 years who were diagnosed with any stroke or transient ischemic attack and recruited during 2007-2010 at 47 stroke centers residing in 14 countries. The study protocol, patient population, and main outcomes were described in detail elsewhere [9-12]. This study was approved by the ethics committee at the leading center (University of Rostock, Rostock, Germany) and in all participating centers according to their national and institutional legislation.

Because m.3243A $>\mathrm{G}$ is the most frequent mtDNA mutation, and the most common mitochondrial cause of stroke-like-episodes disease, we tested patients' DNAs for this specific mutation. Among sifap1 patients with IS $(n=3291)$, we searched for an enriched patient subpopulation according to the following red flags of the mitochondrial diseases associated with $\mathrm{m} .3243 \mathrm{~A}>\mathrm{G}$ mutation: (a) diagnosed cardiomyopathy or (b) presence of two of the following three features: migraine, short stature $(\leq 165 \mathrm{~cm}$ for males and $\leq 155 \mathrm{~cm}$ for females), and diabetes. Unfortunately, deafness/hearing loss was not recorded in the database. Blood samples were stored at $-80{ }^{\circ} \mathrm{C}$ and were available for 238 IS patients who fulfilled the inclusion criteria. Of these, 177 had preexisting cardiomyopathy and the rest had different combinations of migraine, short stature, and diabetes mellitus.

\section{Cell materials and DNA-analysis}

DNA was extracted by routine methodology from blood samples. Previous studies have indicated that the amount of the mutant m.3243A $>$ G mtDNA form decreases by age in leukocytes [13, 14], and therefore in cases in which a suspicion for presence of m.3243A $>$ G mutations arose, we tried to obtain a second sample of urine epithelial cells for mtDNA analysis, as these cells have shown to represent well the overall tissue mutant mtDNA load. The presence of the mutation and quantitation of mtDNA heteroplasmy was performed by PCR-amplification and solid-phase 
minisequencing methodology, as previously described [15]. Solid-phase minisequencing method we used is a very sensitive one, able to detect 1-2\% mutant mtDNA frequencies with high reliability [16].

\section{Magnetic resonance imaging and data collection}

In the sifap1 study, all participating sites were free to use the set of MRI sequences regularly employed at their center for the imaging of stroke, but the study protocol recommended $\mathrm{T}_{2} / \mathrm{PD}$-weighted and/or FLAIR images along with a diffusion-weighted imaging (DWI) sequence as the minimum. Imaging data were collected and interpreted centrally as described elsewhere [9-11]. There was also a mandatory core data set for the collection of demographic and clinical patient characteristics as all patients underwent a detailed evaluation along with all adequate testing. All data were entered to an electronic database. Patients with IS $(n=3291)$ were classified into etiologic subgroups according to the Trial of Org 10,172 in Acute Ischemic Stroke Treatment (TOAST) classification. In addition, clinical and imaging data were reevaluated in detail for those identified as carriers of the main MELAS mutation.

\section{Results}

Four out of the 238 investigated patients, all previously undiagnosed for MELAS, from four different medical centers, were found to carry the heteroplasmic m.3243A $>\mathrm{G}$ mtDNA mutation. Detailed patient data are presented in the Table 1. MELAS mutation load in blood samples was between 4 (patient 1) and $52 \%$ (patient 2) confirming the presence of MELAS main mutation in all 4 patients. The initial TOAST subgroup classification was small-vessel disease (TOAST 3) in three patients and undetermined (TOAST 5) in one (patient 3). All patients were imaged with brain MRI within 2 days of admission. MRI findings were not specific regarding the infarct pattern or the extent of concomitant changes in the brain (Table 1; Fig. 1). Three of four patients demonstrated deep subcortical infarcts (patients 1-3). No infarct was detected in one (patient 4). Apart from an ischemic lesion, one patient also exhibited high signal of the pulvinar thalami bilaterally on T1-weighted MRI (patient 2).

After mtDNA diagnostics, we were able to reach out to two patients (patients 2 and 4). One patient (patient 4) had deafness in addition to already known features and there were several individuals in the family with short stature and deafness. The other patient (patient 2) again had suffered several episodes of lactic acidosis and infection after his stroke and was under investigations for significant cognitive problems. None of the patients had ophthalmoplegia, visual disturbances, or seizures. Additionally, we were able to examine a urine sample from one patient where the mutation load was $85 \%$, higher than in the blood (52\%, patient 2). Unfortunately, the two further patients could not be reexamined.

\section{Discussion}

Testing for the main MELAS mutation in a fairly young stroke population selected on the basis of specific phenotypic features that are suggestive for mitochondrial disease led to positive findings in four patients $(\sim 1.7 \%)$. All these patients went unrecognized for their MELAS-like phenotypic features when they were hospitalized in the acute phase of their ISs. Thus, our study demonstrates that in a phenotype-targeted group, a reasonable number of MELAS mutation carriers can be detected with a rather inexpensive approach. Using a clinical algorithm similar to ours for genetic testing for the m.3243A $>$ G MELAS mutation may well be cost-effective, as the yield for detecting potential cases of MELAS was rather high with modest costs in this study. Mitochondrial disease diagnostics are important not only for the index patient, but also for the family members, at least in terms of genetic counseling, as several unrecognized additional cases could be found multiplying the benefits of diagnostic testing in our patients.

Previous studies reported mitochondrial diseases among consecutive young IS patients to be as low as 2 in 1008 [6] and 6 among 3331 [8], with higher rates (4 among 104) among pediatric patients [7]. Underdiagnosis is potentially possible as all patients in these studies were not systematically tested for mitochondrial diseases and no results of comprehensive testing for mitochondrial diseases in selected or unselected IS patient series are available. MELAS diagnostics in the aforementioned studies were based on clinical suspicion and further genetic counseling, but no systematic searches were performed. A recent study demonstrated that less than half (17/41) of MELAS patients had left ventricular hypertrophy or dysfunction [17], therefore, our selection criteria and results may well be underestimating the number of MELAS patients in the sifap study population. However, since we could not perform full-scale mitochondrial disease diagnostic testing in all our mutation-positive patients, we can only report on mutation-positive IS patients, whereas aforementioned young stroke studies confirmed MELAS diagnosis in their patients with stroke-like episodes [6-8].

MELAS main mutation tested in unselected populations was found to be 7/2954 among white people in one study [18] and 4/3168 in neonatal umbilical cord blood samples from sequential live births in the United Kingdom [19]. Interestingly, all seven individuals in the former study had 
Table 1 Basic characteristics of the 4 ischemic stroke patients diagnosed with MELAS

\begin{tabular}{|c|c|c|c|c|}
\hline & Patient 1 & Patient 2 & Patient 3 & Patient 4 \\
\hline $\begin{array}{l}\text { Age at stroke } \\
\text { onset }\end{array}$ & 46 & 49 & 40 & 46 \\
\hline Gender & Male & Male & Male & Female \\
\hline Family history & Unknown & Nothing significant & $\begin{array}{l}\text { Cardiac and renal } \\
\text { disease }\end{array}$ & Cerebrovascular disease \\
\hline $\begin{array}{l}\text { Symptoms at } \\
\text { admission }\end{array}$ & $\begin{array}{l}\text { Dysarthria, diplopia, } \\
\text { vertigo, hemiparesis }\end{array}$ & Dysarthria, motor and sensory hemiparesis & Diplopia, vertigo & $\begin{array}{l}\text { Vomiting, ataxia, } \\
\text { vertigo, sensory } \\
\text { symptoms }\end{array}$ \\
\hline $\begin{array}{l}\text { mtDNA mutation } \\
\text { load }(\%)\end{array}$ & 4 & 52 & 10 & 41 \\
\hline Echocardiography & Yes & Yes & Yes & Yes \\
\hline $\begin{array}{l}\text { Patent foramen } \\
\text { ovale }\end{array}$ & No & No & No & No \\
\hline Thrombolysis & No & Intravenous thrombolysis & No & No \\
\hline $\begin{array}{l}\text { Medical history } \\
\text { and risk factors }\end{array}$ & $\begin{array}{l}\text { Migraine, diabetes, } \\
\text { smoking, } \\
\text { cardiomyopathy }\end{array}$ & $\begin{array}{l}\text { Coronary disease, myocardial infarction, } \\
\text { coronary bypass operation, hypertension, } \\
\text { snoring, migraine, diabetes }\end{array}$ & $\begin{array}{l}\text { Cardiomyopathy, } \\
\text { smoking, } \\
\text { snoring }\end{array}$ & $\begin{array}{l}\text { Diabetes, migraine, } \\
\text { hypertension, fatigue }\end{array}$ \\
\hline Prior medication & Aspirin, statin & Clopidogrel, ACE inhibitor, statin & None & $\begin{array}{l}\text { Aspirin, ACE inhibitor, } \\
\text { calcium channel } \\
\text { blocker, diuretic, } \\
\text { statin, insulin }\end{array}$ \\
\hline Stature $(\mathrm{cm})$ & 181 & 166 & 183 & 155 \\
\hline Weight $(\mathrm{kg})$ & 95 & 63 & 93 & 59 \\
\hline $\begin{array}{l}\text { Arrival NIHSS } \\
\text { score }\end{array}$ & 9 & 9 & 2 & 3 \\
\hline $\begin{array}{l}\text { TOAST } \\
\text { classification }\end{array}$ & Small-vessel disease & Small-vessel disease & $\begin{array}{l}\text { Undetermined } \\
\text { etiology }\end{array}$ & Small-vessel disease \\
\hline MRI findings & $\begin{array}{l}\text { Small acute left medial } \\
\text { thalamic infarct } \\
\text { extending into the } \\
\text { mesencephalon }\end{array}$ & $\begin{array}{l}\text { Acute infarct in the left thalamus extending into } \\
\text { the posterior limb of the internal capsule. } \\
\text { Bilateral T1 signal hyperintensity of pulvinar } \\
\text { thalami }\end{array}$ & $\begin{array}{l}\text { Old infarcts in } \\
\text { left cerebellum }\end{array}$ & $\begin{array}{l}\text { Few punctate white } \\
\text { matter } \\
\text { hyperintensities, no } \\
\text { acute infarct on DWI }\end{array}$ \\
\hline
\end{tabular}

mild to moderate hearing loss [18]. Another recent study reported that $1 / 400$ were carriers of a MELAS mutation in the United Kingdom [20]. Of the 129 patients reviewed in this report, $28 \%$ had clinical features not consistent with the clinical syndrome and only $9 \%$ of the carriers were asymptomatic [20]. This further indicates that we could have missed additional mutation carrying patients using typical phenotype characteristics to delineate the cohort of 238 patients that was tested. Our mutation carriers are highly likely to have MELAS rather than being carriers only: first, they all appeared with ISs; second, they had major phenotypic features of MELAS; third, we found an incidence of $1.7 \%$ that is well-above that was found in general populations ( $\sim 1$ in 1000) $[18,19]$; and last, individuals carrying MELAS mutation are only rarely asymptomatic [20].

Our findings suggest that positive MELAS main mutation is a considerable finding among young to middle-aged IS patients when they harbor typical phenotypic features for mitochondrial disease. Furthermore, MELAS may also be underdiagnosed even at dedicated stroke centers. A general routine mitochondrial disease genetic search in all young IS patients may not be warranted given its low costeffectiveness, but this has so far not been formally assessed. Considering the experience in general populations [18-20], the number needed to analyze may be as high as 1000. Probably, it is worth testing for the m.3243A $>\mathrm{G}$ mutation in all young to middle-aged patients with IS-like episodes harboring the main clinical features of mitochondrial disease similar to our algorithm as our approach delivered a number needed for an analysis of 60. Because association of other mitochondrial diseases with stroke-like episodes (e.g., MERRF and MIRAS) are based on individual case reports, routine screening for other mitochondrial diseases in unselected IS patient populations or even in young patients is unlikely to be cost-effective. Similar studies could be completed in distinct ethnic populations as our findings are only valid for white European-originating 

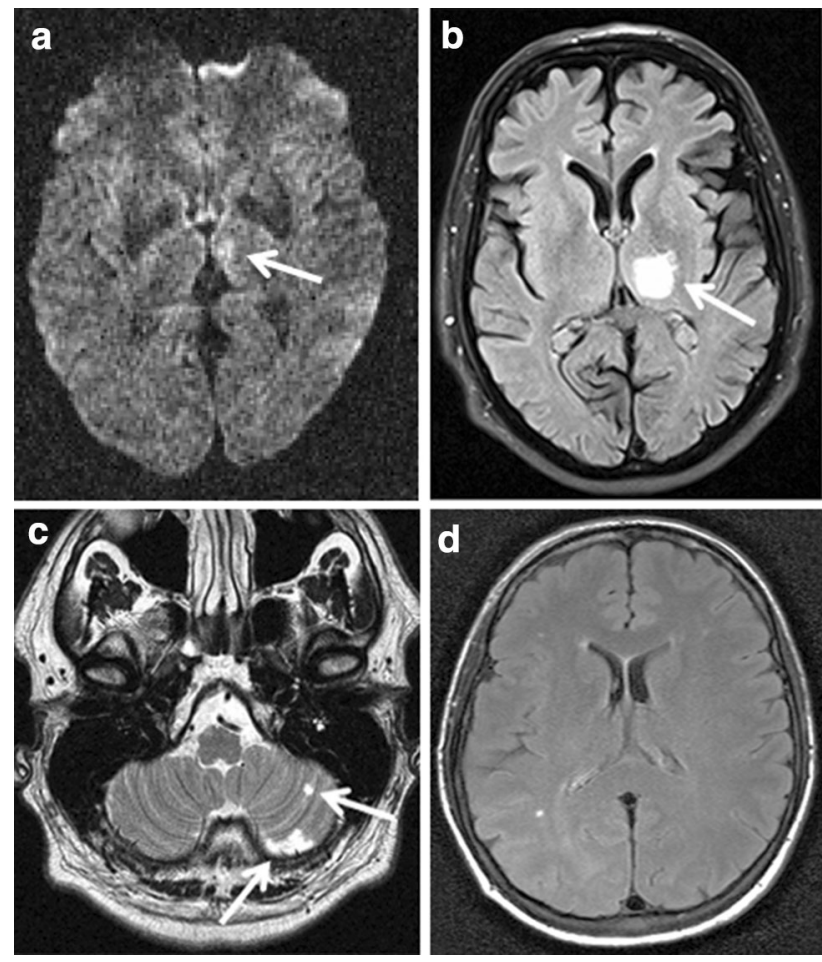

Fig. 1 Magnetic resonance images depicted from all four patients. White arrows indicate infarcts. a Patient 1 with left acute medial thalamic infarct extending into mesencephalon (DWI), b patient 2 with acute infarct in the left thalamus extending into the posterior limb of the internal capsule (FLAIR), c patient 3 demonstrating old infarcts in left cerebellum (T2), and d patient 4 showing few punctate white matter hyperintensities, but no acute infarction on MRI (FLAIR)

individuals. However, whole mtDNA diagnostic testing is unlikely to yield additional information as most other mtDNA abnormalities are not associated with stroke-like episodes. Whole mtDNA testing is rather costly $(\sim 2000 €$ per sample) and requires substantial expert physician time to analyze and interpret the findings.

Whether our MELAS main mutation finding was the cause or a bystander in our patients is not certain, as most MELAS patients developing stroke-like episodes were reported to present with multiple cortical infarct-like changes not confined strictly to arterial territories, a finding which was not seen in our cohort. Instead, we detected deep-territory infarcts in two patients, cerebellar infarction in one patient, and no MRI-visible acute ischemic lesion in one patient. Unfortunately, we could not reevaluate our mutation-positive four patients for a definite MELAS diagnosis. Therefore, a positive MELAS main mutation finding could only be an incidental finding instead of the cause, although potentially an under-recognized atypical pattern of MELAS disease that could easily be overlooked by physicians is possible. A recent article including 11 patients with MELAS and encephalopathy, but without any stroke-like episodes reported deep gray matter lesions in seven of the patients [21]. This supports the view that, indeed, atypical lesions may not be so rare in the absence of typical stroke-like episodes. Obviously, we cannot conclude MELAS as the putative cause in these patients. Initially, three out of four patients were classified as having small-vessel disease and one as having stroke of undetermined etiology according to the TOAST classification by the treating physicians. Thus, mitochondrial disease was not considered in any patient. Notably, despite preexisting cardiomyopathy in two patients, in neither patient was cardiogenic embolism considered a cause of IS.

Strengths of our study are the large source patient population and the well-documented phenotypic characteristics. One of the main limitations, in turn, is the fact that we did not test mitochondrial disease in all study participants and reserved testing to those with phenotypic features for mitochondrial diseases. Therefore, we are not certain whether routine screening of young IS patients for MELAS disease would have yielded additional patients. Moreover, we tested only for the m.3243A $>$ G mutation, which covers approximately $80 \%$ of all MELAS patients [3]. Therefore, our results may still be an underestimation. Furthermore, we could not reach out to two of the four MELAS mutation-positive patients for further scrutiny of family history. Our patient population is also limited to white European patients, which diminishes the generalizability of our findings to other populations.

In conclusion, mtDNA testing for MELAS in a relatively young IS patient population with phenotypic targeting may disclose a considerable number of previously unrecognized MELAS main mutation carrying patients at a reasonable cost. However, it remains uncertain whether mitochondrial disease was the cause of ISs in our 4 patients and to what extent our approach underestimates the overall frequency of MELAS mutations in such populations.

Acknowledgments During this study, AS was funded by Sigrid Jusélius Foundation, Jane and Aatos Erkko Foundation, European Research Council, University of Helsinki, and Academy of Finland. We are grateful to all study investigators and the core facility study team at the University of Rostock.

\section{Compliance with ethical standards}

Funding The sifap1 study (http://www.sifap.eu/, NCT00414583) has been supported by an unrestricted scientific Grant from Shire Human Genetic Therapies to the University of Rostock.

\section{Conflicts of interest None.}

Ethical standards Ethics committee of the Medical Association Mecklenburg-Vorpommern (Board 2), University of Rostock, Rostock, Germany has approved this study on 14-SEPTEMBER-2006. 


\section{References}

1. Ylikallio E, Suomalainen A (2012) Mechanisms of mitochondrial diseases. Ann Med 44:41-59

2. Finsterer J (2006) Central nervous system manifestations of mitochondrial disorders. Acta Neurol Scand 114:217-238

3. Sakuta R, Goto Y, Horai S, Nonaka I (1993) Mitochondrial DNA mutations at nucleotide positions 3243 and 3271 in mitochondrial myopathy, encephalopathy, lactic acidosis, and stroke-like episodes: a comparative study. J Neurol Sci 115:158-160

4. Finsterer J (2009) Management of mitochondrial stroke-likeepisodes. Eur J Neurol 16:1178-1184

5. Al-Hassnan ZN, Rashed MS, Al-Dirbashi OY, Patay Z, Rahbeeni Z, Abu-Amero KK (2008) Hyperornithinemia-hyperammonemiahomocitrullinuria syndrome with stroke-like imaging presentation: clinical, biochemical and molecular analysis. J Neurol Sci 264:187-194

6. Putaala J, Metso AJ, Metso TM, Konkola N, Kraemer Y, Haapaniemi E, Kaste M, Tatlisumak T (2009) Analysis of 1008 consecutive patients aged 15 to 49 with first-ever ischemic stroke: the Helsinki young stroke registry. Stroke 40:1195-1203

7. Salih MA, Abdel-Gader AG, Zahraa JN, Al-Rayess MM, Alorainy IA, Hassan HH, Ruitenbeek W, Zeviani M (2006) Stroke due to mitochondrial disorders in Saudi children. Saudi Med J 27(Suppl 1):S81-S90

8. Yesilot Barlas N, Putaala J, Waje-Andreassen U, Vassilopoulou S, Nardi K, Odier C, Hofgart G, Engelter S, Burow A, Mihalka L, Kloss M, Ferrari J, Lemmens R, Coban O, Haapaniemi E, Maaijwee N, Rutten-Jacobs L, Bersano A, Cereda C, Baron P, Borellini L, Valcarenghi C, Thomassen L, Grau AJ, Palm F, Urbanek C, Tuncay R, Durukan Tolvanen A, van Dijk EJ, de Leeuw FE, Thijs V, Greisenegger S, Vemmos K, Lichy C, Bereczki D, Csiba L, Michel P, Leys D, Spengos K, Naess H, Tatlisumak T, Bahar SZ (2013) Etiology of first-ever ischaemic stroke in European young adults: the 15 cities young stroke study. Eur J Neurol 20:1431-1439

9. Rolfs A, Martus P, Heuschmann PU, Grittner U, Holzhausen M, Tatlisumak T, Bottcher T, Fazekas F, Enzinger C, Ropele S, Schmidt R, Riess O, Norrving B, sifap1 Investigators (2011) Protocol and methodology of the Stroke in Young Fabry Patients (sifap1) study: a prospective multicenter European study of 5,024 young stroke patients aged $18-55$ years. Cerebrovasc Dis 31:253-262

10. Rolfs A, Fazekas F, Grittner U, Dichgans M, Martus P, Holzhausen M, Bottcher T, Heuschmann PU, Tatlisumak T, Tanislav C, Jungehulsing GJ, Giese AK, Putaala J, Huber R, Bodechtel U, Lichy C, Enzinger C, Schmidt R, Hennerici MG, Kaps M, Kessler C, Lackner K, Paschke E, Meyer W, Mascher H, Riess O, Kolodny E, Norrving B, Stroke in Young Fabry Patients (sifap) Investigators (2013) Acute cerebrovascular disease in the young: the Stroke in Young Fabry Patients study. Stroke 44:340-349
11. Fazekas F, Enzinger C, Schmidt R, Dichgans M, Gaertner B, Jungehulsing GJ, Hennerici MG, Heuschmann P, Holzhausen M, Kaps M, Kessler C, Martus P, Putaala J, Ropele S, Tanislav C, Tatlisumak T, Norrving B, Rolfs A, sifap1 Investigators (2013) MRI in acute cerebral ischemia of the young: the Stroke in Young Fabry Patients (sifap1) study. Neurology 81:1914-1921

12. von Sarnowski B, Putaala J, Grittner U, Gaertner B, Schminke U, Curtze S, Huber R, Tanislav C, Lichy C, Demarin V, Basic-Kes V, Ringelstein EB, Neumann-Haefelin T, Enzinger C, Fazekas F, Rothwell PM, Dichgans M, Jungehulsing GJ, Heuschmann PU, Kaps M, Norrving B, Rolfs A, Kessler C, Tatlisumak T, sifap1 Investigators (2013) Lifestyle risk factors for ischemic stroke and transient ischemic attack in young adults in the Stroke in Young Fabry Patients study. Stroke 44:119-125

13. 't Hart LM, Jansen JJ, Lemkes HH, de Knijff P, Maassen JA (1996) Heteroplasmy levels of a mitochondrial gene mutation associated with diabetes mellitus decrease in leucocyte DNA upon aging. Hum Mutat 7:193-197

14. Rahman S, Poulton J, Marchington D, Suomalainen A (2001) Decrease of 3243 A-> $\mathrm{G}$ mtDNA mutation from blood in MELAS syndrome: a longitudinal study. Am J Hum Genet 68:238-240

15. Suomalainen A, Majander A, Pihko H, Peltonen L, Syvanen AC (1993) Quantification of tRNA3243(Leu) point mutation of mitochondrial DNA in MELAS patients and its effects on mitochondrial transcription. Hum Mol Genet 2:525-534

16. Suomalainen A, Syvanen AC (2003) Analysis of nucleotide sequence variations by solid-phase minisequencing. Methods Mol Biol 226:361-366

17. Malfatti E, Laforet P, Jardel C, Stojkovic T, Behin A, Eymard B, Lombes A, Benmalek A, Becane HM, Berber N, Meune C, Duboc D, Wahbi K (2013) High risk of severe cardiac adverse events in patients with mitochondrial m.3243A $>\mathrm{G}$ mutation. Neurology 80:100-105

18. Manwaring N, Jones MM, Wang JJ, Rochtchina E, Howard C, Mitchell P, Sue CM (2007) Population prevalence of the MELAS A3243G mutation. Mitochondrion 7:230-233

19. Elliott HR, Samuels DC, Eden JA, Relton CL, Chinnery PF (2008) Pathogenic mitochondrial DNA mutations are common in the general population. Am J Hum Genet 83:254-260

20. Nesbitt V, Pitceathly RD, Turnbull DM, Taylor RW, Sweeney MG, Mudanohwo EE, Rahman S, Hanna MG, McFarland R (2013) The UK MRC Mitochondrial Disease Patient Cohort Study: clinical phenotypes associated with the m.3243A $>\mathrm{G}$ mutation-implications for diagnosis and management. J Neurol Neurosurg Psychiatry 84:936-938

21. Tschampa HJ, Urbach H, Greschus S, Kunz WS, Kornblum C (2013) Neuroimaging characteristics in mitochondrial encephalopathies associated with the m.3243A $>$ G MTTL1 mutation. J Neurol 260:1071-1080 